\title{
The Individualized Management Approach for Acute Poisoning
}

\author{
Muneera Al-Jelaify (D) ${ }^{1,2}$ and Suliman AlHomidah (iD) \\ ${ }^{1}$ Pharmacy Services, King Khalid University Hospital, King Saud University Medical City, Riyadh, Saudi Arabia \\ ${ }^{2}$ Pediatric Intensive Care Unit, Pediatrics Department, King Khalid University Hospital, King Saud University Medical City, \\ Riyadh, Saudi Arabia \\ Correspondence should be addressed to Muneera Al-Jelaify; maljelaify@ksu.edu.sa
}

Received 23 March 2021; Accepted 30 April 2021; Published 12 May 2021

Academic Editor: Mahmoud S. Ahmed

Copyright ( $) 2021$ Muneera Al-Jelaify and Suliman AlHomidah. This is an open access article distributed under the Creative Commons Attribution License, which permits unrestricted use, distribution, and reproduction in any medium, provided the original work is properly cited.

\begin{abstract}
Acute poisoning is a widespread emergency that mandates early management decisions for optimal outcomes. An individualized approach is an ideal way to provide those outcomes. Promoting awareness among healthcare professionals managing acute poisoning about the importance of incorporating the pharmacokinetics and following certain criteria to consider interventions such as activated charcoal, antidote, or specific investigations may improve their risk assessment strategies and management plans. To address the main aspects that should be considered to develop a customized poisoning management plan, we conducted this review based on relevant publications recovered by a careful search in PubMed. Our opinions as experts from the King Saud University (KSU) Drug and Poison Information Center (DPIC) were considered in the review.
\end{abstract}

\section{Introduction}

Acute poisoning is a widespread emergency situation that mandates early management decisions to safeguard optimal outcomes while at the same time avoids redundant investigation, intervention, or observation. An organized, individualized approach for patient assessment and management is the ideal way to provide the best emergency care for acute poisoning. The objective of this article is to address the main aspects that should be considered to develop a customized care plan for every patient [1].

The heterogeneous nature of patients presented with acute poisoning requires more than an understanding of the ingested substance. To design a rational therapeutic plan, many factors need to be considered: the ingested amount, time since ingestion, clinical presentation, patient factors, geographical site, and available resources. A patient-tailored approach is vital to ensure the best outcomes [1].

\section{Our Poisoning Center and Experience in Poisoning Management}

The Pharmacy in King Khalid University Hospital (KKUH) at King Saud University in the Riyadh area at the Kingdom of Saudi Arabia established the drug and poison information center in October 1983. The main objective to include poison information service was to provide evidence-based, expert advice from clinical pharmacists trained and certified to manage poisoning cases throughout the Kingdom of Saudi Arabia. The service, since its inception until present, offers consultations 24 hours (calls from 7: 30 am to 4:00 pm are managed by the DPIC, while after working hours, consultations are managed by on-call clinical pharmacists). The provided information is targeted towards physicians, eventhough the advice was also available to other paramedical personnel and the common public [2]. 
Our center is experienced in providing optimal, patientspecific care that consider multiple factors. Such that, for any poisoning case regardless of the exposure route or the substance ingested, the general approach for management should be followed, which targets four main areas: initial assessment of airway, breathing and circulation, gastrointestinal decontamination, enhanced elimination, and antidotes. In addition to other specific factors that influence the therapy plan which include the following.

\section{Drug Pharmacokinetics (PKs) in Overdoes [3-17]}

We should always keep in mind that PKs in cases of overdose differ from the usual PKs, so the assumption that the usual PKs interpretation can be utilized for poisoning cases should be carefully implemented, especially for the majority of poisons; the kinetics during toxicity is rarely reported.

3.1. Onset of Action. Onset of action refers to the time required for a drug/agent response to be observed. It can aid to know if the poison action started. So one can anticipate the toxicity signs and symptoms and if antidote administration is considered, which is the best time to administer it, and when its action will start if given.

3.2. Time to Peak. Time to peak is defined as the time it takes for a drug/agent to reach its highest blood concentration. Although there is a rough relationship between the peak concentration of the poison and the occurrence of clinical toxicity, however, time to peak can still be utilized to predict a time window for appearance of signs and symptoms and help to decide about observation time and disposition, especially for drugs with long half-lives.

3.3. Bioavailability. Bioavailability refers to the fraction of the dose that reaches the systemic circulation. It can be affected by increasing the dose because of gastrointestinal (GI) physiology changes, saturation of absorption, or due to the first-pass effect. It can be determined by using the area under the curve (AUC) that presents the systemic exposure to the toxin. It cannot be estimated accurately in poisoning cases.

3.4. Half-Life. Half-life is the time it takes for the concentration of the drug/agent in the blood to be reduced by $50 \%$. It can help to predict the toxic level of the drug/agent and help to decide about observation time and disposition and about the appropriate time for drug/agent levels or other lab tests.

It should not be used solely; rather, other parameters must be considered to decide on management since absorption, distribution, and clearance can change during an overdose. Also, the onset and duration of action in toxic doses may associate poorly with the half-life. It is very commonly seen that pharmacodynamics rely on distribution kinetics rather than clearance.

3.5. Volume of Distribution ( $V d)$. Volume of distribution reflects the distribution of a drug between plasma and the rest of the body. It has an adjunct role in poisoning management where you can use it to decide about dialysis as a toxin's elimination method since drugs with low $\mathrm{Vd}$ are more likely to be dialyzed.

You can also change the Vd by adjusting the fluid status to decrease the concentration in the blood if the ingested substance has a distribution limited to the plasma compartment. Also, it may aid in deciding about observation time and disposition. Developers of the drug nomograms such as paracetamol nomogram estimated the proper time to withdraw the drug level to be after 4 hours, allowing enough time for the drug to be absorbed and then distributed.

Poisonous substances undergoing redistribution or having a large volume of distribution may induce delayed toxicity manifestations because they will take a longer time to be eliminated even when using enhanced elimination strategies because those methods cannot reach through if the poison accumulated in the brain or lung, for example.

3.6. Protein Binding. Protein binding is the degree to which a drug attaches to plasma proteins (albumin, lipoprotein, and glycoprotein). It can magnify the severity of toxicity in the case of hypoalbuminemia for highly albumin-bound substances, since hypoalbuminemia increases the free fraction of the drug in the blood, which increases the risk of toxicity, and can give a clue about the possibility for the ingested substance to undergo dialysis or plasma exchange, where drugs with low protein binding affinity are more likely to be dialyzed, while drugs with high protein binding affinity are more likely to be removed by plasma exchange.

3.7. Metabolism. Define first if the drug is metabolized by the liver or excreted mostly unchanged in the urine. If metabolized, then check whether the metabolites are active, as it will extend the duration of toxicity. Also, if metabolism will reach saturation, it can result in unpredictable toxin's concentration.

Reduction in the first-pass effect can dramatically increase the absorption of oral drugs even with therapeutic doses like in patients with cirrhosis. This change will make the dose and toxicity relationship unpredictable for drugs undergoing significant first-pass effects (e.g., calcium channel blockers).

Using the enzyme induction method to enhance poison elimination is a theory that cannot be utilized in management of acute poisoning where care should be immediate while enzyme induction may take time to work. But reducing the amount of toxic metabolites can help, such as in cases of alcohol poisoning. 
3.8. Excretion. Check first if the poison is eliminated mainly by the kidney in urine or not. If so, the half-life will be affected. This will affect the decisions to utilize enhanced elimination methods, including dialysis or urine alkalinization for weak acid drugs and the observation/disposition time. The longer the half-life, the longer the time the victims may need under observation.

3.9. Pharmacokinetics Role in Dialysis Decision. Knowing the PKs and physicochemical characters of the ingested drug will help decide the applicability of dialysis as a toxin elimination method, if the drug has a small molecular weight and volume of distribution, a low affinity of binding to plasma proteins, and a low endogenous rate of clearance, then the toxin is considered dialyzable. Table 1 presents some of the characteristics with the most applicable removal method $[4,18]$.

Common examples of drugs where toxicity can be severe and dialysis options are considered a therapeutic solution are carbamazepine and phenytoin. Table 2 summarizes the PKs properties of each drug and the likeliness of dialysis efficacy [19-21].

\section{Activated Charcoal (AC) [22-24]}

AC is still considered a universal antidote for acutely poisoned patients, eventhough its use has declined significantly in recent years. This is mainly attributed to it is possible risks, uncertainty about its benefits [25-28], and that clinicians have less interest in GI decontamination as a mode of therapy. The commonly mentioned safety concern is a pulmonary aspiration, but the incidence is still low and increases with risk factors such as vomiting, seizures, and altered mental status. Some very rare adverse events from case reports include GI complications (emesis, esophageal perforation, GI perforation, and intestinal obstruction), pulmonary complications (chronic lung disease, obstructive laryngitis with glottic edema, granulomatous lung mass, charcoal empyema, and bronchiolitis obliterans), and corneal abrasions.

\subsection{Usual Conditions and Requirements to Consider Single- Dose AC.}

The ingested agent can be adsorbed to AC

Severe toxicity is expected

Recent overdose

Conscious and alert patient

Secured airway

The antidote is not available

Ingestion of long-acting agents (i.e., modified, extended, or sustained dosage forms)

No intestinal obstruction or ileus
4.2. Timing of Administration. Several studies concluded that the optimal time for administration of AC is within 1 hour of poison ingestion. However, late administration beyond 1 hour can be considered in some cases, for example, if a large quantity of an agent is administered, which carries a very high risk of morbidity and mortality with very limited therapeutic options. Moreover, it can be used when there is a high chance that the ingested drug/agent remains in the GI tract (i.e., coingestion of drugs that slow gastric emptying or in the presence of food), and using AC might positively impact the patient's outcomes.

This mnemonic (PHAILS) outlines the common agents and conditions for which AC is not generally indicated $[22,25,29-33]$ :

P-Pesticides, Petroleum distillates, un-Protected airway

H-Hydrocarbons, Heavy metals, >1 Hour presentation

A-Acids, Alkali, Alcohols, Altered level of consciousness, Aspiration risk

I-Iron, Ileus, Intestinal obstruction

L-Lithium, Lack of gag reflex

S-Solvents, Seizures

\section{Antidotes, Clinical Pearls [34]}

Value from antidotes is generally time-dependent without certainties. For many victims of poisons, clinical improvement is expected with supportive management only. Most of the poisoning-related mortalities are because of a lack of timely supportive management rather than the absence of antidote administration. Despite the enthusiasm by clinicians to use antidotes, the approved antidotes are around 20 that cannot cover the huge number of drugs and toxins available.

For ideal antidotes use, recognize conditions in which their use could yield clinically significant improvements in morbidity or mortality. If the utility cannot be quantified, estimate the risk from giving the antidote. Many common antidotes are primarily safe (e.g., activated charcoal or vitamins), while some can cause hypersensitivity reactions (e.g., N-acetylcysteine or antivenoms); dosing errors is another risk factor that limits antidote use (e.g., an overdose of flumazenil or naloxone can induce seizures). The toxicity from using antidotes can be precipitated or exaggerated when administered for multiple overdoses (e.g., flumazenil may provoke seizures without recovery from coma in patients with a concomitant antipsychotic overdose).

\section{Investigations and Screening Tests $[1,3]$}

The most commonly recommended screening tests for acute poisoning are ECG and the serum paracetamol level. Drug concentrations are required only for few agents for the purpose of risk assessment and to decide on timely needed interventions (e.g., paracetamol, salicylate, and iron). Other 
TABLe 1: Drug characteristics and the corresponding suitable dialysis method.

\begin{tabular}{lccc}
\hline & Hemodialysis & Hemoperfusion & Hemofiltration \\
\hline Molecular weight & $<500 \mathrm{Da}$ & $<50,000 \mathrm{Da}$ & $<50,000 \mathrm{Da}$ \\
Protein binding & Poorly bound & Low or high & Poorly bound \\
Volume of distribution & $<1 \mathrm{~L} / \mathrm{Kg}$ & $<1 \mathrm{~L} / \mathrm{Kg}$ & $<1 \mathrm{~L} / \mathrm{Kg}$ \\
Solubility & Hydrophilic & Hydrophilic or lipophilic & Hydrophilic \\
Endogenous clearance & $<4 \mathrm{~mL} / \mathrm{Kg} / \mathrm{min}$ & $<4 \mathrm{~mL} / \mathrm{Kg} / \mathrm{min}$ & $<4 \mathrm{~mL} / \mathrm{Kg} / \mathrm{min}$ \\
\hline
\end{tabular}

TABle 2: Carbamazepine and phenytoin PKs and the likeliness of dialysis efficacy.

\begin{tabular}{|c|c|c|c|}
\hline Drug & Usual PKs & PKs in overdose & Efficacy of dialysis \\
\hline Carbamazepine & $\begin{array}{l}\text { (i) Molecular mass: } \\
236 \mathrm{Da} \text { (small) } \\
\text { (ii) Vd: } 2 \mathrm{~L} / \mathrm{kg} \text { (high); } \\
\text { lipophilic } \\
\text { (iii) Protein binding: } \\
70-80 \% \text { (high) } \\
\text { (iv) Initial half-life is } \\
25-65 \mathrm{~h} \text { and then } 10 \mathrm{~h} \\
\text { (v) Active metabolites } \\
\text { (vi) Urine elimination } \\
\text { (72\%) } \\
\text { (vii) Induces its own } \\
\text { metabolism (chronic use) } \\
\text { (viii) Time to peak =4 } \\
\text { hours }\end{array}$ & $\begin{array}{l}\text { (i) Protein binding } \\
\text { does not decrease } \\
\text { significantly in overdose } \\
\text { (ii) No enzyme induction in } \\
\text { CBZ naive individuals } \\
\text { (iii) Half-life much } \\
\text { longer (ongoing } \\
\text { absorption, impaired } \\
\text { elimination, or both) } \\
\text { (iv) High concentration } \\
\text { exhibits anticholinergic } \\
\text { proprieties, which } \\
\text { delay GI motility, further } \\
\text { prolonging absorption, with } \\
\text { time to peak }>100 \text { hours }\end{array}$ & $\begin{array}{l}\text { High-efficiency } \\
\text { hemodialysis is } \\
\text { the best method } \\
\text { Then, venovenous } \\
\text { CRRT and charcoal } \\
\text { or resin hemoperfusion } \\
\text { May reduce the } \\
\text { carbamazepine level by about } 50 \%\end{array}$ \\
\hline Phenytoin & $\begin{array}{l}\text { (i) Molecular mass: } \\
252 \mathrm{Da} \text { (low) } \\
\text { (ii) } \mathrm{Vd}: 0.7 \mathrm{~L} / \mathrm{kg} \text { (low) } \\
\text { (iii) Protein binding: } \\
\text { 90-95\% (high) } \\
\text { (iv) Half-life }=22 \mathrm{~h} \\
\text { (v) } \mathrm{Cl}=23 \mathrm{~mL} / \mathrm{min} \\
\text { (vi) Follows nonlinear } \\
\text { PKs }\end{array}$ & $\begin{array}{l}\text { (i) Clearance reduced } \\
\text { (ii) Half-life greatly } \\
\text { prolonged (103 hours) } \\
\text { (iii) Protein } \\
\text { binding } \\
\text { decreases }(70-80 \%) \\
\text { (iv) Volume } \\
\text { of distribution increases }\end{array}$ & $\begin{array}{l}\text { High-efficiency } \\
\text { hemodialysis is the best } \\
\text { method, but hemoperfusion is an acceptable alternative. } \\
\text { However, because of the low incidence of irreversible tissue } \\
\text { injury or death related to phenytoin poisoning and the } \\
\text { relatively limited effect of dialysis on phenytoin removal, } \\
\text { dialysis can be used only in very selected patients with severe } \\
\text { phenytoin poisoning. }\end{array}$ \\
\hline
\end{tabular}

investigations are requested selectively where the findings support management.

Some of the compelling indications for specific tests in acutely poisoned patients may include the following:

Guide risk assessment or prognosis

Help to include or exclude differential diagnoses, complications, and suspected poisonous agents

Help to decide about the benefit of decontamination methods and/or antidote administration

Monitor the response to provided care and the limitations or endpoint to ongoing interventions

\section{Observation and Admission Criteria [3, 17]}

Judgments related to hospitalization for patients with suspected poisoning are occasionally difficult. The majority of poisoning victims will be asymptomatic, and a short observation time in the emergency room is often the only necessary thing to do.
The type (drug, household products, etc.), nature (e.g., acidic or basic, lipid base or hydrophilic), amount, form (e.g., immediate-release or sustained), pharmacokinetics, and pharmacodynamics of the ingested substance should always be taken into consideration, as it will be very helpful to decide about disposition or admission and the required time for observation and monitoring.

It is very important during the formulation of the caring plan to investigate the nature and history of the poisoning case. Intentional overdose may indicate psychosocial troubles, while an unreliable history in children may point to the likelihood of abuse or neglect. In both situations, you need to admit the child for expert consultations.

\section{Conclusion}

An individualized management approach is an optimal way to provide patient care. Promoting awareness among healthcare professionals managing acute poisoning about the importance of incorporating the pharmacokinetics parameters and following certain criteria to consider 
interventions such as activated charcoal, antidote, or specific investigations or lab tests may improve their risk assessment strategies and management plans.

\section{Conflicts of Interest}

The authors declare that they have no conflicts of interest.

\section{References}

[1] F. F. S. Daly, M. Little, and L. Murray, "A risk assessment based approach to the management of acute poisoning," Emergency Medicine Journal, vol. 23, no. 5, pp. 396-399, 2006.

[2] A. Saddique, "Poisoning in Saudi Arabia: ten-year experience in king khaled university hospital," Archive of Annals of Saudi Medicine, vol. 21, no. 1-2, pp. 88-91, 2001.

[3] M. Riordan, G. Rylance, and K. Berry, "Poisoning in children 1: general management," Archives of Disease in Childhood, vol. 87 , no. 5, pp. 392-396, 2002.

[4] D. M. Roberts and N. A. Buckley, "Pharmacokinetic considerations in clinical toxicology," Clinical Pharmacokinetics, vol. 46, no. 11, pp. 897-939, 2007.

[5] J. Rosenberg, N. L. Benowitz, and S. Pond, "Pharmacokinetics of drug overdose," Clinical Pharmacokinetics, vol. 6, no. 3, pp. 161-192, 1981.

[6] D. Platt, "Pharmacokinetics of drug overdose," Clinics in Laboratory Medicine, vol. 10, no. 2, pp. 261-269, 1990.

[7] D. A. Gorelick, "Pharmacokinetic strategies for treatment of drug overdose and addiction," Future Medicinal Chemistry, vol. 4, no. 2, pp. 227-243, 2012.

[8] G. K. Isbister, "Pharmacokinetic-pharmacodynamic modeling in overdose patients - is it worth the trouble?" Clinical Toxicology, vol. 48, no. 9, pp. 896-897, 2010.

[9] M. Edscape, "What are the pharmacokinetics of phenytoin toxicity," 2021, https://www.medscape.com/answers/816447168669/what-are-the-pharmacokinetics-of-phenytoin-toxicity.

[10] I. Fernando, "Predicting serum drug level using the principles of pharmacokinetics after an overdose: a case of lithium overdose," Australasian Psychiatry, vol. 25, no. 4, pp. 391-394, 2017.

[11] J. M. Cooper, S. B. Duffull, A. S. Saiao, and G. K. Isbister, "The pharmacokinetics of sertraline in overdose and the effect of activated charcoal," British Journal of Clinical Pharmacology, vol. 79, no. 2, pp. 307-315, 2015.

[12] M. Levine and A.-M. Ruha, "Overdose of atypical antipsychotics," CNS Drugs, vol. 26, no. 7, pp. 601-611, 2012.

[13] M. Edscape, "Oral hypoglycemic agent toxicity treatment \& management," 2020, https://emedicine.medscape.com/ article/1010629-treatment.

[14] H. A. Spiller and T. S. Sawyer, "Toxicology of oral antidiabetic medications," American Journal of Health-System Pharmacy, vol. 63, no. 10, pp. 929-938, 2006.

[15] E. W. Boyer, "Management of opioid analgesic overdose," New England Journal of Medicine, vol. 367, no. 2, pp. 146-155, 2012.

[16] I. L. Frithsen and W. M. Simpson, "Recognition and management of acute medication poisoning," American Family Physician, vol. 81, no. 3, pp. 316-323, 2010.

[17] Decision Support in Medicine, "Pharmacology and toxicology: treatment of poisons - general description of problem," 2021.

[18] E. Aibek, "The role of renal replacement therapy in the management of pharmacologic poisonings," International Journal of Nephrology, vol. 3, Article ID 3047329, 2016.
[19] M. Ghannoum, C. Yates, T. F. Galvao et al., "Extracorporeal treatment for carbamazepine poisoning: systematic review and recommendations from the EXTRIP workgroup," Clinical Toxicology, vol. 52, no. 10, pp. 993-1004, 2014.

[20] K. Anseeuw, J. B. Mowry, E. A. Burdmann et al., "Extracorporeal treatment in phenytoin poisoning: systematic review and recommendations from the EXTRIP (extracorporeal treatments in poisoning) workgroup," American Journal of Kidney Diseases, vol. 67, no. 2, pp. 187-197, 2016.

[21] S. Takki, "Pharmacokinetic evaluation of hemodialysis in acute drug overdose," Journal of Pharmacokinetics and Biopharmaceutics, vol. 6, no. 5, pp. 427-442, 1978.

[22] R. M. Lapus, "Activated charcoal for pediatric poisonings: the universal antidote?" Current Opinion in Pediatrics, vol. 19, no. 2, pp. 216-222, 2007.

[23] D. N. Juurlink, "Activated charcoal for acute overdose: a reappraisal," British Journal of Clinical Pharmacology, vol. 81, no. 3, pp. 482-487, 2016.

[24] T. Zellner, "The use of activated charcoal to treat intoxications," Archive of Deutsches Ärzteblatt International, vol. 116, pp. 311-317, 2019.

[25] American Academy of Clinical Toxicology, "European association of poisons centres and clinical toxicologists. Position paper: single-dose activated charcoal," Clinical Toxicology, vol. 43, no. 2, pp. 61-87, 2005.

[26] K. S. Merigian, "Prospective evaluation of gastric emptying in the self-poisoned patient," American Journal of Emergency Medicin, vol. 8, p. 479, 1990.

[27] K. Kulig, "Management of acutely poisoned patients without gastric emptying," Annals of Emergency Medicine, vol. 14, p. 562, 1985.

[28] S. M. Pond, "Gastric emptying in acute overdose: a prospective randomized controlled trial," Medical Journal of Australia, vol. 163, p. 345, 1995.

[29] M. Eddleston, "Multiple-dose activated charcoal in acute selfpoisoning: a randomized controlled trial," Lancet, vol. 371, no. 9612, pp. 579-587, 2008.

[30] M. Eddleston, "Management of acute organophosphorus pesticide poisoning," Lancet, vol. 371, no. 9612, pp. 597-607, 2008.

[31] G. K. Isbister and V. V. Kumar, "Indications for single-dose activated charcoal administration in acute overdose," Current Opinion in Critical Care, vol. 17, no. 4, pp. 351-357, 2011.

[32] P. Gaudreault, "Activated charcoal revisited," Clinical Pediatric Emergency Medicine, vol. 6, pp. 76-80, 2005.

[33] B. Bailey, "To decontaminate or not to decontaminate? The balance between potential risks and foreseeable benefits," Clinical Pediatric Emergency Medicine, vol. 9, no. 1, pp. 17-23, 2008.

[34] N. A. Buckley, "Who gets antidotes? Choosing the chosen few," British Journal of Clinical Pharmacology, vol. 81, no. 3, pp. 402-407, 2016. 